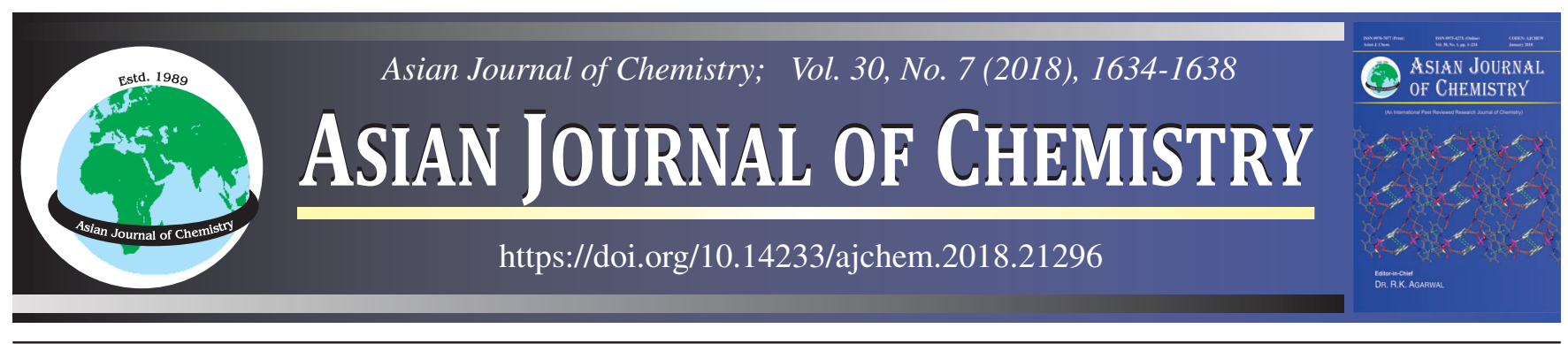

\title{
One-Pot, Green Approach Synthesis of 2-Aryl Substituted Benzimidazole Derivatives Catalyzed by Water Extract of Papaya Bark Ash
}

\section{K. Kantharaju* and Prashant B. Hiremath}

Department of Chemistry, Peptide and Medicinal Chemistry Research Laboratory, Rani Channamma University, Belagavi-591 156, India

*Corresponding author: E-mail: kk@rcub.ac.in

Received: 20 February 2018;

Accepted: 20 April 2018;

Published online: 31 May 2018;

AJC-18945

A simple, green, rapid and environmentally benign route has been developed for the synthesis of 2-substituted benzimidazoles by the reaction of substituted $o$-phenylenediamine with different substituted benzoyl chloride using water extract of papaya bark ash (WEPBA) as a green catalyst at room temperature. This method provides several added advantages of being completely green, economic, giving high yields and minimizing use of hazardous solvents. Further, the separated product does not required any kind of chromatographic purification.

Keywords: Green synthesis, Benzimidazole, $o$-Phenylenediamine, Benzoyl chloride, Water extract of papaya bark ash.

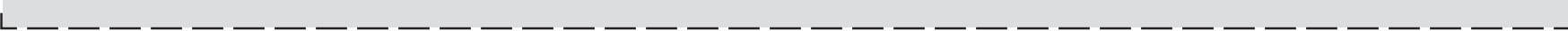

\section{INTRODUCTION}

Development of alternative method that use environmentally benign solvents and biodegradable, non-toxic chemical alternative to pollution and toxic chemicals for the organic reaction are emerging in recent years as a green protocol in organic synthesis [1].

Recently, use of agro waste stuff [2] as chemical surrogates has become one of the most emerging in research endeavours for organic chemists. Researchers also reported several agro waste stuff extracted were employed for many organic syntheses and observed successful alternative replacement of many toxic and environmentally causing solvent. Some of the recent reported extracts are water extract of papaya bark ash (WEPBA) [3], water extract of banana peel ash (WEBPA) [4], water extract of rice straw ash (WERSA) [5], etc. These sustainable technologies protect environment and human health by an economically safer approach. It focuses pollution free, environmentally friendly design on production and use of alternative chemical solvent in economical feasibility. However, use of solvent remain a constant source of concern in organic synthesis as it gives toxic and hazardous in storing and waste solvent disposal issues. Solvents are still preferred on account of their easy separation and evaporation, which helps in extraction and separation of many compound which are thermally sensitive reactants. Therefore, in recent years the chemical synthesis based solvent free or neat condition [6] is the best alternative for these issues, as the replace of toxic solvent to simple water extract makes a protocol green.
Benzimidazole and its derivatives found in a variety of naturally occuring compounds and structurally similar to purine bases [7]. It is well documented that benzimidazole and its derivatives extensively possesing wide range of spectrum of biological activities [8]. These compounds showed selective non-peptide LH-RH antagonist [9], lymphocyte specific kinase inhibitor [10], N-methyl-D-asparatate kinase inhibitor [11], N-methylD-aspartate antagonist [12], 5-lipoxygenase inhibitor [13], neuropeptide N-S5B polymerase inhibitor, neuropeptide YY1 receptor antagonist [14], thrombin inhibitors [15] and poly (ADP-ribose) polymerase inhibitors [16], DNA-miner groovebinding agents possess antitumour activity [17], topoisomerase inhibitors, angiotensin II inhibitors and proloferartion inhibitors [18]. In addition, various promising therapeutic applications of benzimidazole of derivatives found in antimicrobial [19], anticancer [20], antiulcer, antifungal [21], antihypertensive [22], herbicides [23], antihistamine activity [24] and other veterinary applications [25]. Further, benzimidazole scaffolds showed significant activity against several viruses such as RNA [26], influenza [27], human cytomegalovirus [28], HIV [29], herpes (HSV-1) [30], selective angiotensin II inhibitors [31] and 5HIJ antagonists [32]. In addition, benzimidazoles also play key intermediates and serve as ligands for stereospecific catalysis synthesis [33]. Due to this broad spectrum of benzimidazole derivative importance in biological applications has given much attention for the synthesis of benzimidazole and its derivatives. The most common method for the preparation of benzimidazole derivative involves the condensation of $o$-phenylenediamine 
and carbonyl of aldehyde or acid derivatives. The condensation of $o$-phenylenediamine with carboxylic acid carried out in the presence of strong acidic conditions and high temperature [34, 35]. The other method involves oxidative cyclodehydrogenation of Schiff bases [36], generated from $o$-phenylenediamine with aldehydes in the presence of various catalysts [37]. The various catalysts employed are $\mathrm{K}_{3} \mathrm{PO}_{4}$ [38], CAN [39], oxone [40], $\mathrm{DDQ}$ [41], $\mathrm{I}_{2}$ [42], $\mathrm{PhI}(\mathrm{OAc})_{2}$ [6] and $\mathrm{KHSO}_{4}$ [43]. In addition, several other catalysts such as metal halides [44-46], metal oxychloride [47,48], ionic liquid [49], heteropoly acids [50], BDSB [51], L-proline solid-supported catalyst [52], polymer supported catalyst [53] and microwave assisted have also been reported in the literature [54-57]. Nevertheless, many of these methods used expensive and toxic reagents with elevated temperature reactions, several condition leads to isolation of lesser yield, tedious work-up and impure compound isolation. As a consequence, the introduction of simple, eco-friendly and faster reaction rate is still required to overcome some of these limitations. As a part of developing newer application towards organic synthesis using greener catalyst employed for the product formation of amide using WEBPA, Knoevenagel condensation [58], coumarins [59] synthesis catalyzed by water extract of papaya bark ash (WEPBA), herein, the simple and ecofriendly synthesis of 2-aryl benzimidazole derivatives catalyzed by water extract of papaya bark ash at room temperature as a green method protocol.

\section{EXPERIMENTAL}

All the reagents were purchased from commercial sources and used as received without further purification. Melting points were determined in open capillaries and are uncorrected. FTIR spectra were recorded in $\mathrm{KBr}$ pellets on a Shimadzu, ${ }^{1} \mathrm{H}$, ${ }^{13} \mathrm{C}$ NMR spectra were recorded on a Bruker 300 spectrometer using TMS as an internal standard. HR-MS spectra were recorded in MALDI-TOF mass spectrometer. The progress of the reaction monitored by TLC, yields referred to isolated pure products.

Preparation of catalyst water extract of papaya bark ash: The water-extract of papaya bark ash was prepared by burning dried papaya bark to ashes. Then, wieghed $10 \mathrm{~g}$ of ash and suspended in $100 \mathrm{~mL}$ distilled water and stirred for $2 \mathrm{~h}$ at room temperature. The suspension was then filtered and a light yellow coloured extracted was termed as WEPBA. The $\mathrm{pH}$ of the soluton is found to be 11.54 .

General procedure for the synthesis of 2-aryl substituted benzimidazoles: To a mixture of $o$-phenylenediamine ( $1 \mathrm{mmol}$ ) and benzoyl chloride ( $1 \mathrm{mmol}), 3 \mathrm{~mL}$ of WEPBA added and the contents stirred at room temperature until TLC indicated complete consumption of $o$-phenylenediamine. The completion of the reaction was followed by TLC using hexane:ethyl acetate (7:3) as eluent. After completion of the reaction, 2-substituted benzimidazoles precipitated was filtered, product isolated with no need for any further chromatographic purification. The synthesized benzimidazole derivatives were characterized by comparing with reported m.p., ${ }^{1} \mathrm{H}$ NMR, ${ }^{13} \mathrm{C}$ NMR and HR-MS data.

\section{Spectral data of some selected compounds}

2-Phenyl-1H-benzo[d] imidazole (Entry 1): IR $\left(v_{\max }, \mathrm{cm}^{-1}\right.$, $\mathrm{KBr}): 3420,3047,2920,1623,1450 ;{ }^{1} \mathrm{H}$ NMR $(300 \mathrm{MHz}$, DMSO- $\left.d_{6}\right): \delta 7.2(\mathrm{~d}, 1 \mathrm{H}, \mathrm{Ar}), 7.38-7.46(\mathrm{~m}, 3 \mathrm{H}, \mathrm{Ar}), 7.51-7.53$ (m, 4H, Ar), 7.6-7.63 (m, 4H, Ar), 7.91-7.93 (m, 1H, Ar), 9.94 (bs, $1 \mathrm{H}, \mathrm{NH}) ;{ }^{13} \mathrm{C}$ NMR (100 MHz, DMSO- $\left.d_{6}\right): \delta 124.5,126.1$, 127.5, 128, 132.5, 133, 134.5, 135, 136.1, 138; MS (m/z): 195.09 $\left(\mathrm{M}^{+}\right)$(cal: 194.23).

2-(4-Nitrophenyl)-1H-benzo[d] imidazole (Entry 2): IR $\left(v_{\max }, \mathrm{cm}^{-1}, \mathrm{KBr}\right): 3236,3024,1649,1473 ;{ }^{1} \mathrm{H} \mathrm{NMR}(300 \mathrm{MHz}$, DMSO- $\left.d_{6}\right): \delta$ 7.31-7.33 (m, 4H, Ar), 7.75-7.77 (d, 2H, Ar), 8.11-8.12 (d, 2H, Ar), 11.72 (s, 1H, NH); ${ }^{13} \mathrm{C} \mathrm{NMR} \mathrm{(100} \mathrm{MHz,}$ DMSO- $\left.d_{6}\right): \delta 116.5,119.4,122.3,123.6,124.1,124.7,130.2$, 132.1, 133.3, 135.7, 138.6, 145.8, 150.2; MS ( $\mathrm{m} / \mathrm{z}): 240\left(\mathrm{M}^{+}\right)$ (cal: 239.23).

2-(3-Nitrophenyl)-1H-benzo[d]imidazole (Entry 5): IR $\left(v_{\max }, \mathrm{cm}^{-1}, \mathrm{KBr}\right): 3264,3037,1638,1455 ;{ }^{1} \mathrm{H}$ NMR $(300 \mathrm{MHz}$, DMSO- $\left.d_{6}\right): \delta 7.32-7.35(\mathrm{~m}, 2 \mathrm{H}, \mathrm{Ar}), 7.70-7.73(\mathrm{~m}, 3 \mathrm{H}, \mathrm{Ar})$, 8.01-8.03 (d, 1H, Ar), 8.08-8.10 (d, 1H, Ar), 8.43 (t, 1H, Ar), 11.97 (s, 1H, NH); ${ }^{13} \mathrm{C}$ NMR (100 MHz, DMSO (300 MHz, DMSO- $\left.d_{6}\right): \delta 116.8,117.4,122.1,123.3,123.7,124.1,130.3$, 132.5, 135.1, 137.4, 137.9, 148.1, 149.2; MS (m/z): $240\left(\mathrm{M}^{+}\right)$ (cal: 239.23).

2-(2-Nitrophenyl)-1H-benzo[d]imidazole (Entry 6): IR $\left(v_{\max }, \mathrm{cm}^{-1}, \mathrm{KBr}\right): 3475,3127,1645,1453 ;{ }^{1} \mathrm{H} \mathrm{NMR}(300 \mathrm{MHz}$, DMSO- $\left.d_{6}\right): \delta$ 7.37-7.41 (m, $\left.2 \mathrm{H}, \mathrm{Ar}\right), 7.55-7.68(\mathrm{~m}, 4 \mathrm{H}, \mathrm{Ar})$, 7.91-7.93 (d, 1H, Ar), 8.31-8.32 (d, 1H, Ar), 12.46 (bs, 1H, NH); ${ }^{13} \mathrm{C}$ NMR (100 MHz, DMSO- $\left.d_{6}\right): \delta 116.7,12.2,123.3,123.6$, 124.4, 125.7, 129.6, 134.6, 135.8, 138.1, 139.9, 146.3, 150.4; MS ( $\mathrm{m} / \mathrm{z}): 240\left(\mathrm{M}^{+}\right)$(cal: 239.23).

2-(4-Methylphenyl)-1H-benzo[d]imidazole (Entry 9): IR ( $\left.v_{\max }, \mathrm{cm}^{-1}, \mathrm{KBr}\right): 3279,3022,1634,1471 ;{ }^{1} \mathrm{H}$ NMR $(400$ MHz, DMSO- $\left.d_{6}\right): \delta 1.23$ (s, $\left.3 \mathrm{H}, \mathrm{CH}_{3}\right), 7.20-7.38(\mathrm{~m}, 3 \mathrm{H}, \mathrm{Ar})$, 7.51-7.65 (m, 2H, Ar), 8.05-8.07 (d, 2H, Ar), 8.30 (s, 1H, Ar), $12.87(\mathrm{bs}, 1 \mathrm{H}, \mathrm{NH}) ;{ }^{13} \mathrm{C}$ NMR $\left(100 \mathrm{MHz}\right.$, DMSO- $\left.d_{6}\right): \delta 21.22$, 111.3, 118.9, 121.5, 126.8, 127.8, 127.7, 129.9, 134.9, 139.9, 143.9, 151.6; MS (m/z): $210\left(\mathrm{M}^{+}\right)$(cal: 208.26).

2-(4-Hydroxyphenyl)-1H-benzo[d]imidazole (Entry 10): IR ( $\left.v_{\max }, \mathrm{cm}^{-1}, \mathrm{KBr}\right): 3467,3358,3054,1643,1461 ;{ }^{1} \mathrm{H}$ NMR (400 MHz, DMSO- $d_{6}$ ): $\delta 6.96-6.99$ (d, 2H, Ar), 7.31-7.33 (m, $2 \mathrm{H}, \mathrm{Ar}$ ), 7.66-7.68 (d, 2H, Ar), 7.62-7.68 (m, 2H, Ar), 8.59 (s, $1 \mathrm{H}, \mathrm{OH}), 12.29$ (bs, $1 \mathrm{H}, \mathrm{NH}) ;{ }^{13} \mathrm{C}$ NMR (100 MHz, DMSO- $\left.d_{6}\right)$ : $\delta 115.9,116.1,116.9,122.2,124.2,125.1,126.7,136.2,138.3$, 150.2, 160.3. MS (m/z): $211\left(\mathrm{M}^{+}\right)$(cal: 210.23).

\section{RESULTS AND DISCUSSION}

Herein, we have employed one step synthesis of benzimidazole derivatives from the reaction of $o$-phenylenediamine/ substituted $o$-phenylenediamines with substituted benzoyl chloride. We first optimized the amount of WEPBA required for the reaction by choosing model reaction of $o$-phenylenediamine $(1 \mathrm{mmol})$ and benzoyl chloride $(1 \mathrm{mmol})$ in varied volume of WEPBA from $1 \mathrm{~mL}$ to $5 \mathrm{~mL}$ at room temperature with constant stirring. The progress of the reaction was monitored by TLC. After completion of the reaction, 2substituted benzimidazoles precipitated as a solid, filtered, washed with $2 \mathrm{~mL}$ of $\mathrm{CH}_{3} \mathrm{CN}$ and product obtained found not required further purification. Table-1 summarized the reaction time and physical constant obtained for the isolated products. The best yield observed with 3-5 mL use of WEPBA, lesser the amount of catalytic medium (1 or $2 \mathrm{~mL}$ ) gave lesser yields. Hence, $3 \mathrm{~mL}$ of WEPBA solution is used in the entire syntheses. 
TABLE-1

CHEMICAL STRUCTURE AND PHYSICAL PARAMETERS OF 2-ARYL SUBSTITUTED BENZIMIDAZOLES

\begin{tabular}{|c|c|c|c|c|c|c|c|}
\hline \multirow{2}{*}{ Entry } & \multirow{2}{*}{ Diamine } & \multirow{2}{*}{ Acyl chloride } & \multirow{2}{*}{ Product* } & \multirow{2}{*}{$\begin{array}{l}\text { Time } \\
\text { (min) }\end{array}$} & \multirow{2}{*}{$\begin{array}{l}\text { Yield** } \\
(\%)\end{array}$} & \multicolumn{2}{|c|}{ m.p. $\left({ }^{\circ} \mathrm{C}\right)$} \\
\hline & & & & & & Found & Lit. \\
\hline 1 & & & & 35 & 89 & $292-294$ & $290-293$ \\
\hline 2 & & & & 45 & 89 & $324-326$ & $322-323$ \\
\hline 3 & & & & 50 & 88 & $235-237$ & 238 \\
\hline 4 & & & & 45 & 87 & $140-142$ & $139-141$ \\
\hline 5 & & & & 55 & 88 & $205-207$ & 204-206 \\
\hline 6 & & & & 40 & 83 & $263-265$ & $264-265$ \\
\hline 7 & & & & 45 & 86 & $232-233$ & 234 \\
\hline 8 & & & & 40 & 89 & $177-179$ & $179-180$ \\
\hline 9 & & & & 50 & 81 & $260-262$ & $264-265$ \\
\hline
\end{tabular}




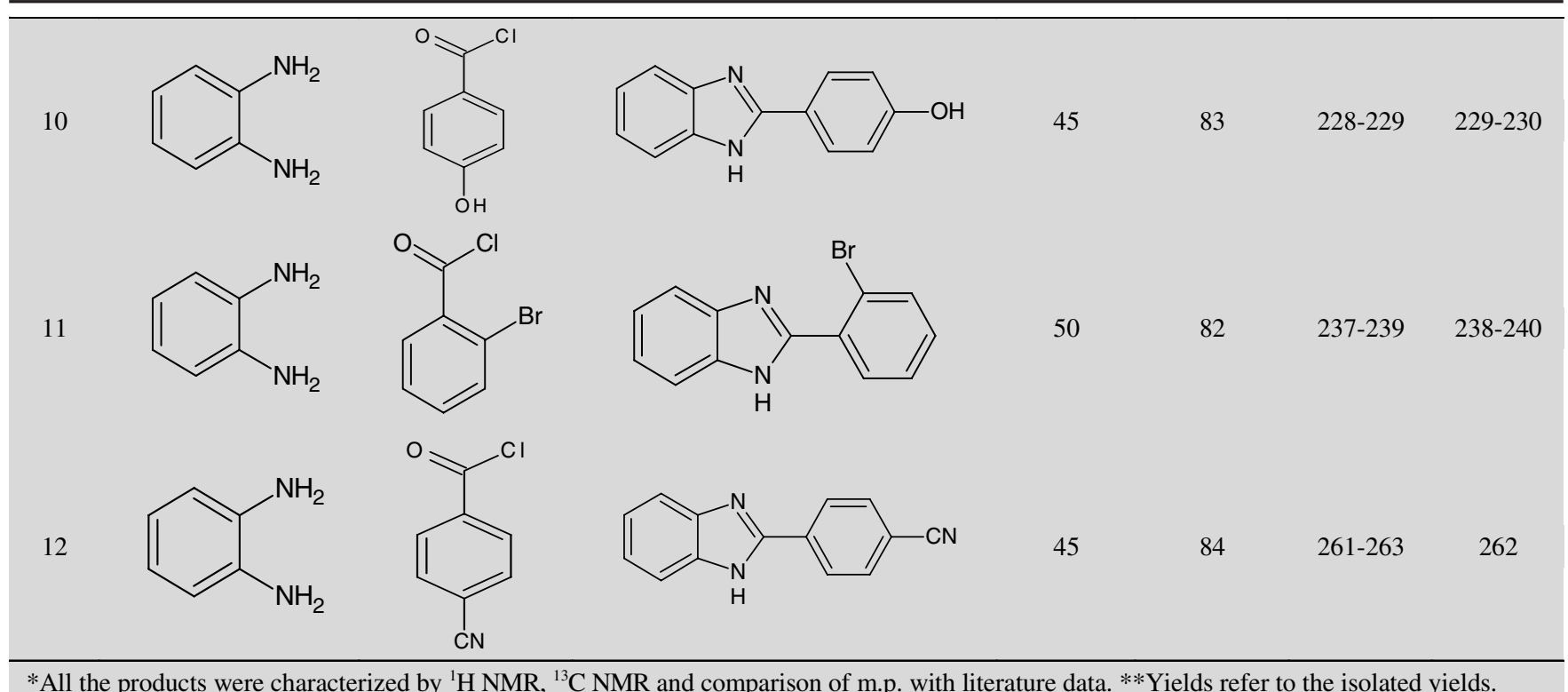

*All the products were characterized by ${ }^{1} \mathrm{H}$ NMR, ${ }^{13} \mathrm{C}$ NMR and comparison of m.p. with literature data. **Yields refer to the isolated yields.

TABLE-2

COMPARISON OF GREEN CATALYST WEPBA EFFICIENCY WITH OTHER RECENTLY REPORTED CATALYST FOR THE SYNTHESIS OF 2-PHENYLBENZIMIDAZOLE

\begin{tabular}{|c|c|c|c|c|c|c|}
\hline Entry & Catalyst & Solvent & Temperature & Time & Yield (\%) & Ref. \\
\hline 1 & Heteropolyacids & $\mathrm{AcOH}$ & Reflux & $4 \mathrm{~h}$ & $92.8-98.2$ & [38] \\
\hline 2 & HZSM & Dioxane (dry) & $100^{\circ} \mathrm{C}$ & $3 \mathrm{~h}$ & 81 & [39] \\
\hline 3 & $\mathrm{BF}_{3}-\mathrm{Et}_{2} \mathrm{O}$ & Dioxane (dry) & $130^{\circ} \mathrm{C}$ & $1-2.5 \mathrm{~h}$ & 97 & [40] \\
\hline 4 & $\mathrm{KF} / \mathrm{Al}_{2} \mathrm{O}_{3}$ & Acetonitrile (dry) & RT & $1 \mathrm{~h}$ & 93 & [41] \\
\hline 5 & {$[\mathrm{bbim}] \mathrm{BF}_{4}$} & Neat & RT & $40 \mathrm{~min}$ & 92 & [42] \\
\hline 6 & {$[\mathrm{Hbim}] \mathrm{BF}_{4}$} & Neat & RT & $10 \mathrm{~min}$ & 95 & [42] \\
\hline 7 & WEPBA & Neat & RT & $35 \mathrm{~min}$ & 96 & This work \\
\hline
\end{tabular}

We compared our novel catalytic method reported here with the literature methods reported for the synthesis of benzimidazole derivative using substituted benzoyl chloride and $o$ phenylenediamine derivative (Table-2). The reported methods are employed in the presence of expensive catalysts like heterogenous [60], zeolites [61], $\mathrm{BF}_{3} \cdot \mathrm{Et}_{2} \mathrm{O}$ [62], $\mathrm{KF} / \mathrm{Al}_{2} \mathrm{O}_{3}$ [63], $[\mathrm{Hbim}] \mathrm{BF}_{4}[64]$ catalysts and organic solvents with elevated temperature, run with longer reaction time. We have chosen substituted benzoyl chlorides for the coupling with $o$-phenylenediamine and found reaction progress without use of any organic solvent and reaction proceeds at room temperature with constant stirring. The product was precipitated in the reaction mixture after simple work-up resulted pure compound isolation without further need of purification. The reaction was found to be greener approach with faster reaction and isolation of very good yield.

\section{Conclusion}

A greener, milder, eco-friendly and benign synthesis of 2 -substituted benzimidazole derivatives using $o$-phenylenediamine and various substituted benzoyl chlorides in the presence of green catalyst water extract of papaya bark ash (WEPBA) at room temperature is developed. The isolated products are found to be pure and not required furthur purification. The homogeniety of the products were examined by using by ${ }^{1} \mathrm{H}$, ${ }^{13} \mathrm{C}$ NMR and mass spectrometry.

\section{ACKNOWLEDGEMENTS}

The authors acknowledge UGC-MRP \{F.43-181/2014 (SR)\}, VGST-SMYSR, DST-FIST for financial support and Prof. Dr. H.N. Gopi, Indian Institute of Science Education and Research, Pune, India for spectral analysis.

\section{REFERENCES}

1. P.J. Walsh, H. Li and C.A. de Parrodi, Chem. Rev., 107, 2503 (2007); https://doi.org/10.1021/cr0509556.

2. M. Sarmah, M. Mondal and U. Bora, Chem. Select, 2, 5180 (2017); https://doi.org/10.1002/slct.201700580.

3. M. Sarmah, A. Dewan, M. Mondal, A.J. Thakur and U. Bora, RSC Adv., 6, 28981 (2016); https://doi.org/10.1039/C6RA00454G.

4. M. Konwar, A.A. Ali and D. Sarma, Tetrahedron Lett., 57, 2283 (2016); https://doi.org/10.1016/j.tetlet.2016.04.041.

5. B. Saikia and P. Borah, RSC Adv., 5, 105583 (2015); https://doi.org/10.1039/C5RA20133K.

6. L.H. Du and P.A. Luo, Synth. Commun., 40, 2880 (2010); https://doi.org/10.1080/00397910903340629.

7. S. Dinesh, G. Shikha, G. Bhavana, S. Nidhi and S. Dileep, J. Pharm. Scient. Innov., 2, 29 (2012).

8. Y. Radha, A. Manjula, B.M. Reddy and B.V. Rao, Indian J. Chem., 50B, 1762 (2011).

9. M. Tatsuta, M. Kataoka, K. Yasoshima, S. Sakakibara, Y. Shogase, M. Shimazaki, T. Yura, Y. Li, N. Yamamoto, J. Gupta and K. Urbahns, Bioorg. Med. Chem. Lett., 2, 2265 (2005); https://doi.org/10.1016/j.bmcl.2005.03.030. 
10. M. Sabat, J.C. VanRens, M.J. Laufersweiler, T.A. Brugel, J. Maier, A. Golebiowski, B. De, V. Easwaran, L.C. Hsieh, R.L. Walter, M.J. Mekel, A. Evdokimov and M.J. Janusz, Bioorg. Med. Chem. Lett., 16, 5973 (2006); https://doi.org/10.1016/j.bmcl.2006.08.132.

11. L. Sun, D. Chiu, D. Kowal, R. Simon, M. Smeyne, R.S. Zukin, J. Olney, R. Baudy and S. Lin, J. Pharmacol. Exp. Ther, 310, 563 (2004); https://doi.org/10.1124/jpet.104.066092.

12. I. Borza, É. Bozó, G. Barta-Szalai, C. Kiss, G. Tárkányi, Á. Demeter, T. Gáti, V. Háda, S. Kolok, A. Gere, L. Fodor, J. Nagy, K. Galgóczy, I. Magdó, B. Ágai, J. Fetter, F. Bertha, G.M. Keserü, C. Horváth, S. Farkas, I. Greiner and G. Domány, J. Med. Chem., 50, 901 (2007); https://doi.org/10.1021/jm060420k.

13. S.A. Smith and R.E. Markwell, Benzimidazoles as 5-Lipoxygenase Inhibitors, US Patent 4925853 (1990).

14. B.G. Stanley, W. Magdalin, A. Seirafi, M.M. Nguyen and S.F. Leibowitz, Peptides, 13, 581 (1992); https://doi.org/10.1016/0196-9781(92)90093-I.

15. E.A. Nutescu, N.L. Shapiro and A. Chevalier, Cardiol. Clin., 26, 169 (2008); https://doi.org/10.1016/j.ccl.2007.12.005.

16. T.D. Penning, G.D. Zhu, J. Gong, S. Thomas, V.B. Gandhi, X. Liu,Y. Shi, V. Klinghofer, E.F. Johnson, C.H. Park, E.H. Fry, C.K. Donawho, D.J. Frost, F.G. Buchanan, G.T. Bukofzer, L.E. Rodriguez, V. BontchevaDiaz, J.J. Bouska, D.J. Osterling, A.M. Olson, K.C. Marsh, Y. Luo and V.L. Giranda, J. Med Chem., 53, 3142 (2010); https://doi.org/10.1021/jm901775y.

17. Y. Kubota, T. Iwamoto and T. Seki, Nucleic Acids Symp. Ser, 42, 53 (1999); https://doi.org/10.1093/nass/42.1.53.

18. H.M. Elokdah, S.Y. Chai and T.S. Sulkowski, US Patent 5764473 (1998); Chem. Abstr., 129, 58.784g (1998).

19. G.A. Kilcigil and N. Altanlar, Turk. J. Chem., 30, 223 (2006).

20. Maruthamuthu, S. Rajam and C.R. Stella, World J. Pharm. Res., 4, 1853 (2015).

21. S. Rajam, C.R. Stella and B.R. Venkatraman, J. Chem. Pharm. Res., 4, 2988 (2012).

22. S. Chandhrasekar, S. Rajam, C.R. Stella and Maruthamuthu, J. Chem. Pharm. Res., 4, 4937 (2012).

23. P.F. Bocion, C.J. Cattanach, P. Eggenberg, J. Gressel, M.-L. Hagmann, S. Malkin and J. Wenger, Pesticide Biochem. Physiol., 28, 75 (1987); https://doi.org/10.1016/0048-3575(87)90115-5.

24. J.-M. Yang and C.-C. Chen, Proteins, 55, 288 (2004); https://doi.org/10.1002/prot.20035.

25. A.A. Spasov, I.N. Yozhitsa, L.I. Bugaeva and V.A. Anisimova, Pharm. Chem. J., 33, 232 (1999); https://doi.org/10.1007/BF02510042.

26. H. Nakano, T. Inoue, N. Kawasaki, H. Miyataka, H. Matsumoto, T. Taguchi, N. Inagaki, H. Nagai and T. Satoh, Bioorg. Med. Chem., 8, 373 (2000); https://doi.org/10.1016/S0968-0896(99)00291-6.

27. N.H. Hauel, H. Nar, H. Priepke, U. Ries, J. Stassen and W. Wienen, J. Med. Chem., 45, 1757 (2002); https://doi.org/10.1021/jm0109513.

28. Y. He, B. Wu, J. Yang, D. Robinson, L. Risen, R. Ranken, L. Blyn, S. Sheng and E.E. Swayze, Bioorg. Med. Chem. Lett., 13, 3253 (2003); https://doi.org/10.1016/S0960-894X(03)00661-9.

29. A.R. Porcari, R.V. Devivar, L.S. Kucera, J.C. Drach and L.B. Townsend, J. Med. Chem., 41, 1252 (1998); https://doi.org/10.1021/jm970559i.

30. T. Roth, M.L. Morningstar, P.L. Boyer, S.H. Hughes, R.W. Buckheit and C.J. Michejda, J. Med. Chem., 40, 4199 (1997); https://doi.org/10.1021/jm970096g.

31. M.T. Migawa, J.-L. Girardet, J.A. Walker, G.W. Koszalka, J.C. Drach, S.D. Chamberlain and L.B. Townsend, J. Med. Chem., 41, 1242 (1998); https://doi.org/10.1021/jm970545c.

32. J. Mann, A. Baron, Y. Opoku-Boahen, E. Johansson, G. Parkinson, L.R. Kelland and S. Neidle, J. Med. Chem., 44, 138 (2001); https://doi.org/10.1021/jm000297b.

33. A. Figge, H.J. Altenbach, J.D. Brauer and P. Tielmann, Tetrahedron Asymm., 13, 137 (2002); https://doi.org/10.1016/S0957-4166(02)00079-4.

34. T. Hisano, M. Ichikawa, K. Tsumoto and M. Tasaki, Chem. Pharm. Bull. (Tokyo), 30, 2996 (1982); https://doi.org/10.1248/cpb.30.2996.

35. B.V.S. Kumar, S.D. Vaidya, R.V. Kumar, S.B. Bhirud and R.B. Mane, Eur. J. Med. Chem., 41, 599 (2006); https://doi.org/10.1016/j.ejmech.2006.01.006.
36. R. Katla, R. Chowrasia, P.S. Manjari and N.L.C. Domingues, RSC Adv., 5, $41716(2015)$; https://doi.org/10.1039/C4RA16222F.

37. I. Bhatnagar and M.V. George, Tetrahedron, 24, 1293 (1968); https://doi.org/10.1016/0040-4020(68)88080-9.

38. K. Bahrami, M.M. Khodaei and F. Naali, J. Org. Chem., 73, 6835 (2008); https://doi.org/10.1021/jo8010232.

39. N. Zheng, K.W. Anderson, X. Huang, H.N. Nguyen and S.L. Buchwald, Angew. Chem., 46, 7509 (2007); https://doi.org/10.1002/anie.200702542.

40. P. Beaulieu, B. Haché and E. von Moos, Synthesis, 1683 (2003); https://doi.org/10.1055/s-2003-40888.

41. J.J. Vanden Eynde, F. Delfosse, P. Lor and Y. van Haverbeke, Tetrahedron, 51, 5813 (1995) https://doi.org/10.1016/0040-4020(95)00252-4.

42. L.-H. Du and Y.-G. Wang, Synthesis, 675 (2007) https://doi.org/10.1055/s-2007-965922.

43. P. Gogoi and D. Konwar, Tetrahedron Lett., 47, 79 (2006); https://doi.org/10.1016/j.tetlet.2005.10.134.

44. M.P. Singh, S. Sasmal, W. Lu and M.N. Chatterjee, Synthesis, 1380 (2000); https://doi.org/10.1055/s-2000-7111.

45. X. Wang, L. Zhang, Y. Xu, D. Krishnamurthy and C.H. Senanayake, Tetrahedron Lett., 45, 7167 (2004); https://doi.org/10.1016/j.tetlet.2004.07.042.

46. A.B. Alloum, K. Bougrin and M. Soufiaoui, Tetrahedron Lett., 44, 5935 (2003); https://doi.org/10.1016/S0040-4039(03)01387-X.

47. D. Yang, H. Fu, L. Hu, Y. Jiang and Y. Zhao, J. Org. Chem., 73, 7841 (2008); https://doi.org/10.1021/jo8014984.

48. S.C. Subramanyam and S. Narayanan, Int. J. Appl. Biol. Pharm. Technol., 1, 689 (2010).

49. S. Lin and L. Yang, Tetrahedron Lett., 46, 4315 (2005); https://doi.org/10.1016/j.tetlet.2005.04.101.

50. R.N. Nadaf, S.A. Siddiqui, T. Daniel, R.J. Lahoti and K.V. Srinivasan, J. Mol. Catal. A, 214, 155 (2004); https://doi.org/10.1016/j.molcata.2003.10.064.

51. M.M. Heravi, S. Sadjadi, H.A. Oskooie, R.H. Shoar and F.F. Bamoharram, Catal. Commun., 9, 504 (2008); https://doi.org/10.1016/j.catcom.2007.03.011.

52. B. Das, H. Holla and Y. Srinivas, Tetrahedron Lett., 48, 61 (2007); https://doi.org/10.1016/j.tetlet.2006.11.018.

53. B. Zou, Q. Yuan and D. Ma, Angew. Chem., 46, 2598 (2007); https://doi.org/10.1002/anie.200700071.

54. R. Varala, A. Nasreen, R. Enugala and R.S. Adapa, Tetrahedron Lett., 48, 69 (2007); https://doi.org/10.1016/j.tetlet.2006.11.010.

55. C.-H. Wu and C.-M. Sun, Tetrahedron Lett., 47, 2601 (2006); https://doi.org/10.1016/j.tetlet.2006.02.015.

56. M.P. Surpur, P.R. Singh, S.B. Patil and S.D. Samant, Synth. Commun., 37, 1375 (2007); https://doi.org/10.1080/00397910701230170.

57. Z. Mao, Z. Wang, J. Li, X. Song and Y. Luo, Synth. Commun., 40, 1963 (2010); https://doi.org/10.1080/00397910903219328.

58. K. Kantharaju and P.B. Hiremath, Int. J. Eng. Technol. Sci. Res., 4, 807 (2017).

59. K. Kantharaju and S.Y. Khatavi, Int. J. Eng. Technol. Sci. Res., 4, 510 (2017).

60. J.Y. Hao, Z.Y. Ge and S.Y. Yang, Synth. Commun., 33, 79 (2003); https://doi.org/10.1081/SCC-120015562.

61. N.J. Coville and E.W. Neuse, J. Org. Chem., 42, 3485 (1977); https://doi.org/10.1021/jo00442a007.

62. V.K. Tandon and M. Kumar, Tetrahedron Lett., 45, 4185 (2004); https://doi.org/10.1016/j.tetlet.2004.03.117.

63. S.B. Khalili and A.R. Sardarian, Monatsh. Chem., 143, 841 (2012); https://doi.org/10.1007/s00706-011-0647-7.

64. S. Neidle, E.L. Rayner, I.J. Simpson, N.J. Smith, J. Mann, A. Baron, Y. OpokuBoahen, K.R. Fox, J.A. Hartley and L.R. Kelland, Chem. Commun., 929 (1999); https://doi.org/10.1039/a901074b.

65. K. Bougrin, A. Loupy and M. Soufiaoui, Tetrahedron, 54, 8055 (1998); https://doi.org/10.1016/S0040-4020(98)00431-1. 